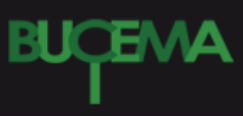

Bulletin du centre d'études médiévales d'Auxerre | BUCEMA

Hors-série $n^{\circ} 8 \mid 2015$

Au seuil du cloître : la présence des laïcs (hôtelleries, bâtiments d'accueil, activités artisanales et de services) entre le Ve et le XII ${ }^{\mathrm{e}}$ siècle

\title{
Les « Hôtelleries Saint-Hugues » de l'abbaye de Cluny
}

Sylvain Aumard, Charlotte Gaillard et Sandra Piotrowski

\section{OpenEdition}

\section{Journals}

Édition électronique

URL : https://journals.openedition.org/cem/13655

DOI : $10.4000 /$ cem.13655

ISSN : 1954-3093

Éditeur

Centre d'études médiévales Saint-Germain d'Auxerre

Référence électronique

Sylvain Aumard, Charlotte Gaillard et Sandra Piotrowski, « Les « Hôtelleries Saint-Hugues » de l'abbaye de Cluny ", Bulletin du centre d'études médiévales d'Auxerre | BUCEMA [En ligne], Hors-série n 8 | 2015 mis en ligne le 28 janvier 2015, consulté le 02 mars 2023. URL : http://journals.openedition.org/cem/ 13655 ; DOI : https://doi.org/10.4000/cem. 13655

Ce document a été généré automatiquement le 2 mars 2023

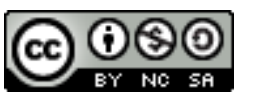

Creative Commons - Attribution - Pas d'Utilisation Commerciale - Partage dans les Mêmes Conditions 4.0 International - CC BY-NC-SA 4.0

https://creativecommons.org/licenses/by-nc-sa/4.0/ 


\title{
Les « Hôtelleries Saint-Hugues » de l'abbaye de Cluny
}

\author{
Sylvain Aumard, Charlotte Gaillard et Sandra Piotrowski
}

Les «Hôtelleries Saint-Hugues", plus connues sous le nom d'«Écuries SaintHugues » et occupées aujourd'hui par théâtre de la ville de Cluny, bordent les places du Marché et du 11 août 1944. Elles sont traditionnellement attribuées à Hugues de Semur (1049-1109) et auraient été édifiées durant la grande campagne de construction de Cluny III. Situé à proximité de la porte d'entrée de

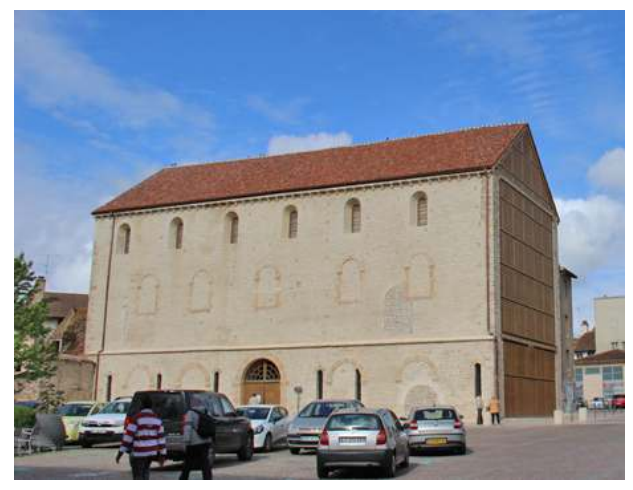
l'ancienne abbaye, l'édifice ouvrait selon un plan anonyme du XVIII ${ }^{\mathrm{e}}$ siècle sur une grande cour intérieure, bordée au sud des hospices de Pierre le Vénérable, au nord de la galilée clunisienne, et enfin à l'est du palais du pape Gélase, par lequel on accédait ensuite au cloître monastique (fig. 1). Par sa position centrale, cette cour desservait ainsi un ensemble de bâtiments destinés à l'accueil des laïcs. 
Fig. 1 - Les Hôtelleries Saint-Hugues vues de l'est (cl. S. Piotrowski).

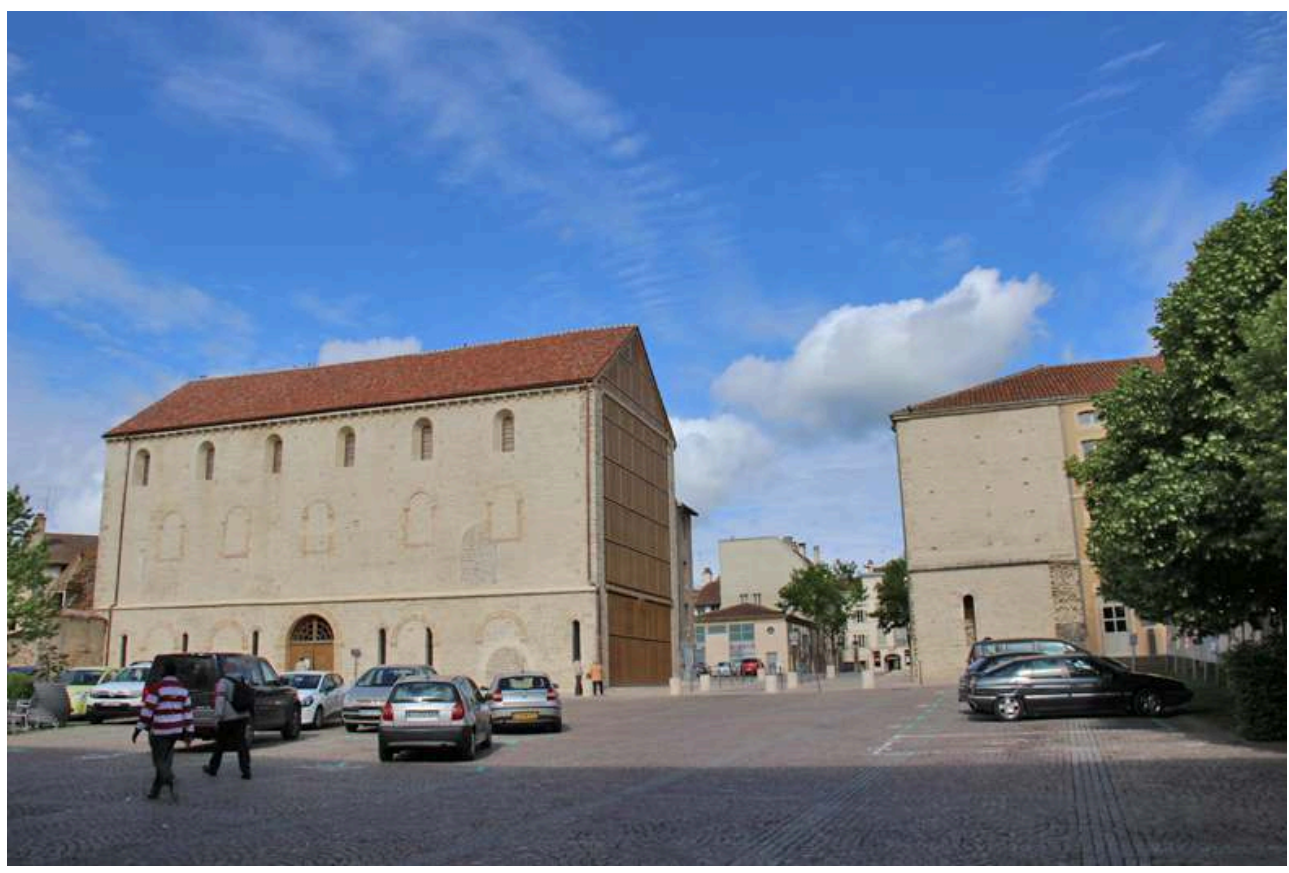

2 Dominique Iogna-Prat a mis en évidence la position toute spécifiquement clunisienne relative à cet accueil au sein des monastères ${ }^{1}$. En effet, l'injonction bénédictine d'accueillir chacun « comme le Christ » s'accommode mal avec la clôture monastique. Or, Cluny se démarque par la place privilégiée accordée aux laïcs, illustrée dès ses débuts par la charte de fondation du monastère. De même, Anne Baud et Gilles Rollier ont relevé cette place spécifique dans le Liber Tramitis, qui dénombre pour l'hôtellerie soixante-dix lits, dont trente réservés aux femmes, fait là encore original ${ }^{2}$. En outre, un réfectoire commun leur est adjoint, tandis que les bancs et les sièges de l'hôtellerie sont ornés de tentures lors des grandes fêtes célébrées dans le monastère.

3 Les restaurations entreprises en 2012 sur l'édifice clunisien avaient pour objectif de réhabiliter l'édifice médiéval, en partie détruit lors de la création de la rue du Marché, s'appuyant en cela sur les observations archéologiques. De plus, elles visaient à une meilleure compréhension de la charpente médiévale, réputée être la plus ancienne de Bourgogne ${ }^{3}$. Elles ont offert l'occasion de s'interroger sur la restitution d'un édifice, qui n'avait fait l'objet finalement que de peu d'investigations jusqu'alors, hormis la solide reconstitution proposée par Kenneth John Conant lors de son travail sur l'abbaye ${ }^{4}$. À l'analyse archéologique du bâti, fondée notamment sur le relevé intégral des maçonneries- mur gouttereau oriental, pignon sud -, s'est donc greffée l'étude de la charpente encore d'origine ${ }^{5}$. Par ailleurs, ce travail s'appuie également sur les nombreux sondages réalisés au pied de l'édifice par Didier Méhu et Nadine Roiné, ainsi que sur un plan relevé par le CEM en 2010 lors de la réfection d'une canalisation. Leurs conclusions sont ici reprises et intégrées à la réflexion d'ensemble ${ }^{6}$.

4 Lors de ses travaux à Cluny, Kenneth John Conant a eu l'occasion de réfléchir sur l'édifice, dont il propose une restitution en élévation, une coupe transversale et une vue axonométrique ${ }^{7}$ (fig. 2). Il s'appuie pour cela sur un plan anonyme de 1700 (fig. 3) et sur le dénombrement de 1623 réalisé par Jacques Veny d'Arbouse, ainsi que sur les données textuelles recueillies dans le Liber Tramitis. 
Fig. 2 - Restitution des Hôtelleries Saint-Hugues proposées par K. J. Conant (fonds Conant).

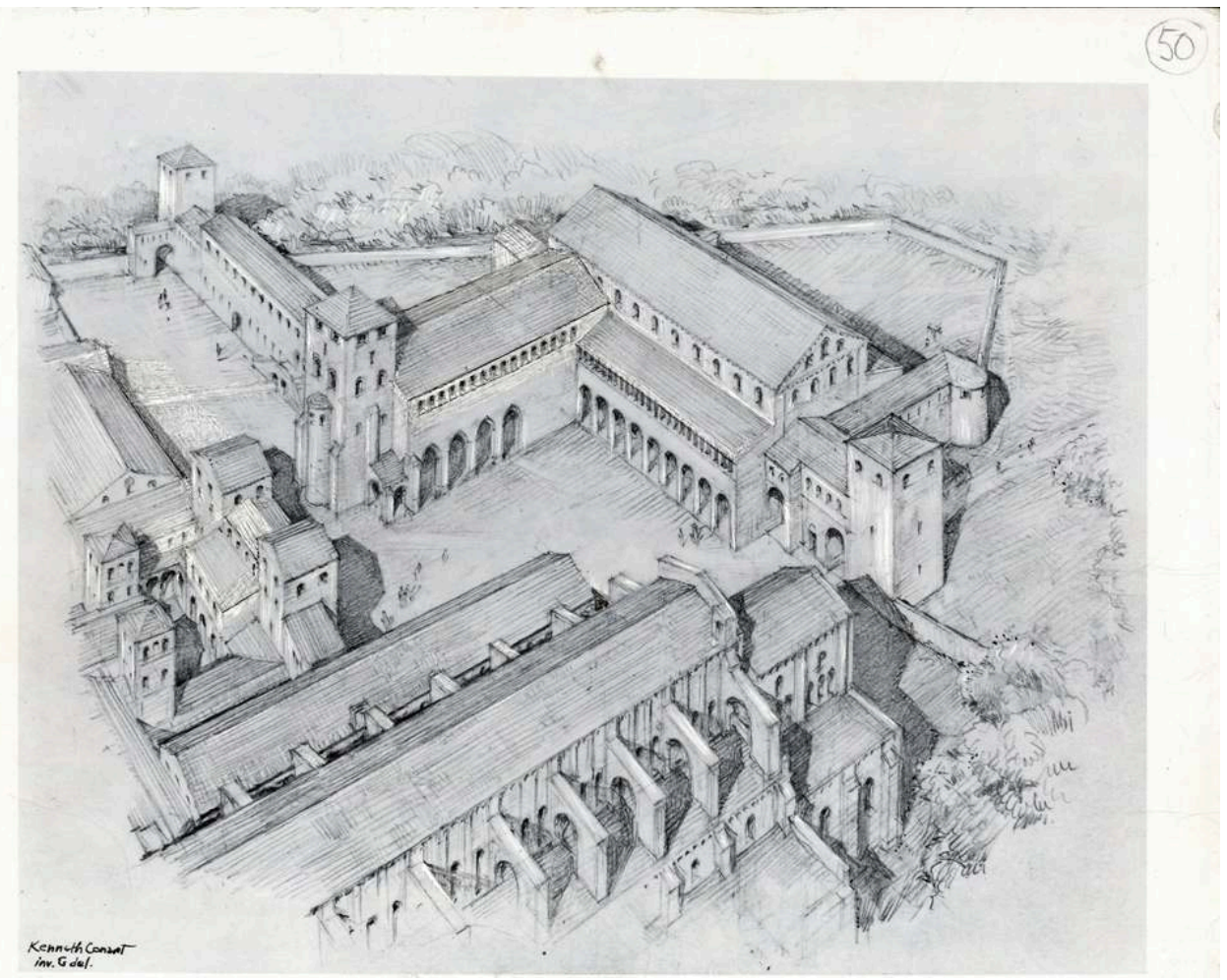

Fig. 3 - Plan anonyme, 1700 (dessin de M. N. Baudrand).

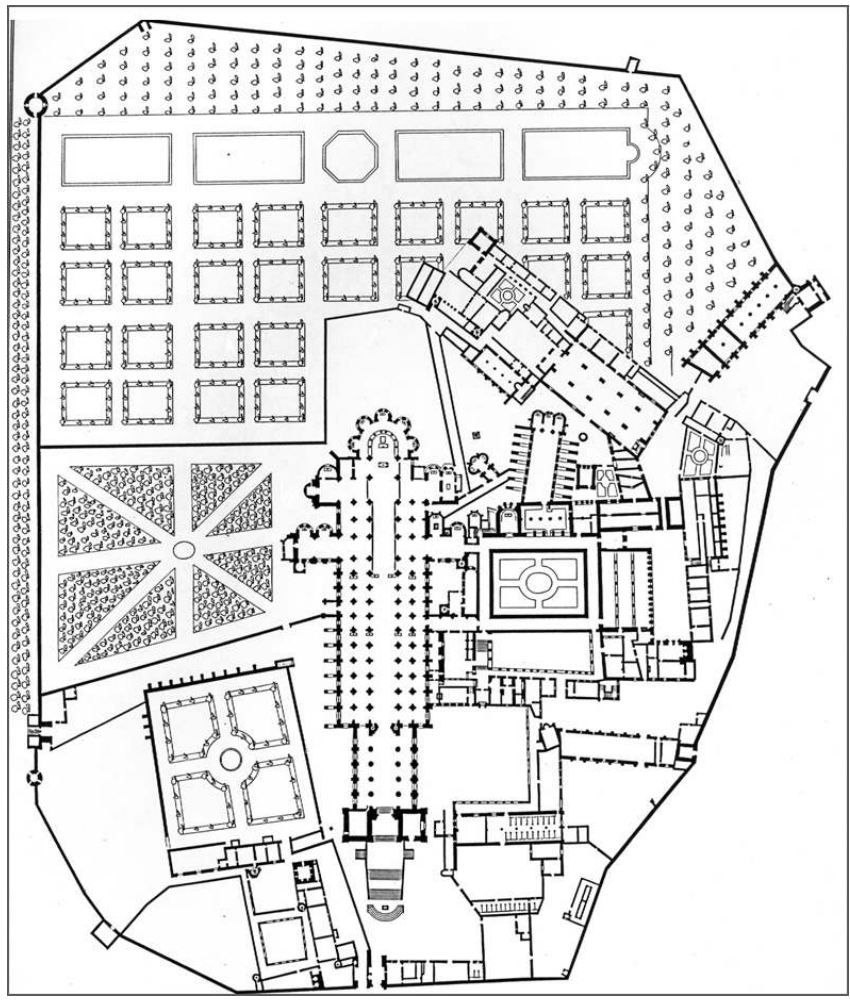

5 Ses carnets révèlent de nombreux croquis, coupes et relevés métrés de l'édifice, très précis. Il décrit notamment avec minutie les dispositions originales de la charpente, ce qui démontre qu'il y a certainement eu accès. Par ailleurs, il note dans les combles la 
présence de décors, dont un orné d'une fleur, sur la corniche de la salle. En revanche, aucun sondage n'a été pratiqué à proximité. D'après ses restitutions, l'édifice se compose de deux niveaux : un rez-de-chaussée percé de petites baies, dont certaines sont encore visibles, et un étage éclairé d'un registre d'ouvertures en plein cintre qui se poursuit sur les pignons nord et sud. De plus, deux galeries flanquent à l'origine les murs gouttereaux est et ouest, ce que révèlent des portes en plein cintre situées à l'étage et ouvrant aujourd'hui sur le vide. Construites sur deux niveaux, elles sont formées d'une façade en pans de bois surmontant une arcature en pierre. Néanmoins, Kenneth John Conant fait part de ses doutes quant à l'édification réelle de la galerie occidentale, prévue mais probablement jamais réalisée.

\section{La restitution de l'édifice après l'analyse archéologique} 8

6 Le théâtre actuel forme un édifice de plan rectangulaire de 29,70 m de long par 11,25 m de large (dans œuvre) et $14,75 \mathrm{~m}$ de haut (fig. 4). Plus au nord, à proximité des tours Barabans, la salle Victor Duruy conserve en partie une baie et les fondations de ce même édifice. Le percement de la rue du Marché au XIX siècle a en effet engendré la division des Hôtelleries en deux bâtiments distincts, qui à l'origine, mesurait donc $52 \mathrm{~m}$ de long.

Fig. 4 - Restitution des Hôtelleries Saint-Hugues d'après l'étude archéologique (dessin S. Aumard, C. Gaillard et S. Piotrowski, d'après équipe de fouille).

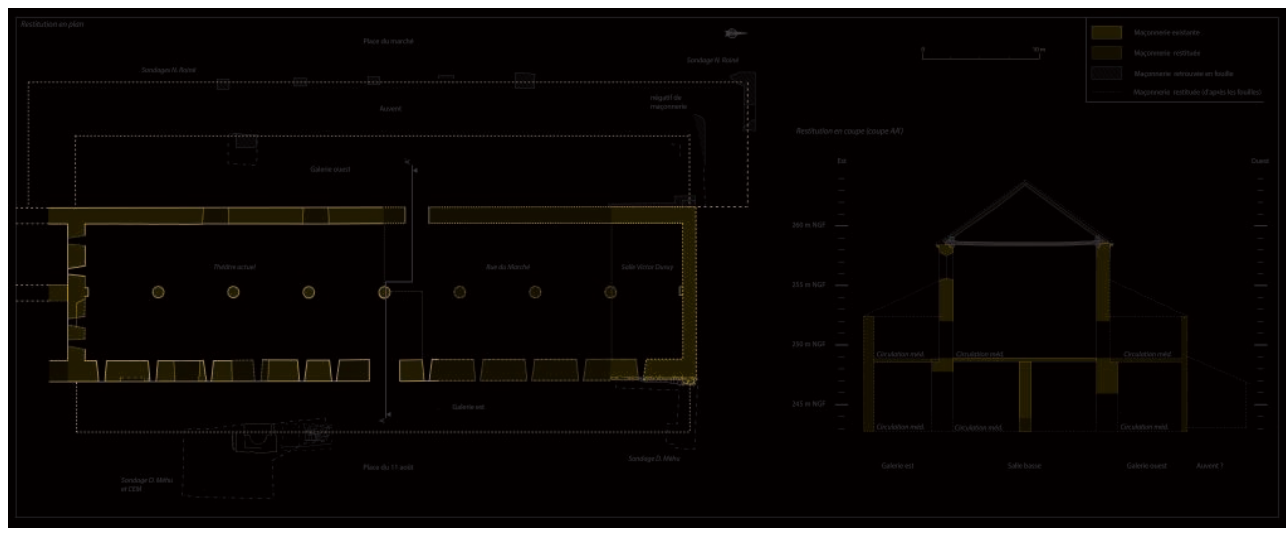

L'édifice s'organise sur deux niveaux. La salle basse, non voûtée, est plafonnée depuis l'origine. Deux vaisseaux de $5 \mathrm{~m}$ de large environ sont séparés d'une colonnade formée d'au moins quatre piles circulaires de $1 \mathrm{~m}$ de diamètre, sur lesquelles repose une double rangée de poutres maîtresses. Avec les muraillères intégrées dans les gouttereaux, ces poutres forment un dispositif assurant l'assise nécessaire au plancher, tout en réduisant la portée des solives ${ }^{9}$. Quant à la salle haute, elle s'élève sur $10 \mathrm{~m}$ de haut environ, surmontée d'une charpente dont le faîte se situe encore $5 \mathrm{~m}$ au-dessus : elle occupe donc un volume de grande ampleur (plus de $7000 \mathrm{~m}^{3}$ !), qui en traduit toute l'importance. D'après le plan anonyme, la salle basse était divisée en deux pièces au xvIII ${ }^{\mathrm{e}}$ siècle : la première au sud comportait des boxes à chevaux et servait manifestement d'écuries, composant le théâtre actuel amputé d'une petite portion; quant à la seconde, au nord, sa fonction n'a pu être précisée. Le dessin indique la présence d'un deuxième pilastre saillant au nord; en revanche, le mur interne n'en 
comporte qu'un seul, mais de dimension beaucoup moins importante. Ce détail pourrait laisser entendre que le niveau inférieur ne constitue à l'origine qu'une seule pièce, divisée en deux dans un second temps, peut-être au moment de la mise en place de l'écurie. Pour ce qui touche les dispositions internes de la salle haute, les aménagements du théâtre actuel empêchent toute observation pertinente.

L'analyse des maçonneries révèle une construction bien intégrée dans le grand chantier de construction de Cluny III, observé sur le transept, le mur gouttereau nord de l'abbatiale et enfin les tours Barabans ${ }^{10}$. Les moellons sont équarris et leur face dressée; ils sont disposés en assises régulières, dont la mise en œuvre annonce la construction en petit appareil (fig. 5). Ils sont taillés selon trois modules, similaires à ceux utilisés sur le transept et le mur gouttereau nord de la nef : longueurs comprises entre 10 et $50 \mathrm{~cm}$ pour des hauteurs épaisses $(15-22 \mathrm{~cm})$, moyennes $(11-14 \mathrm{~cm})$ et fines (6-10 cm). De nombreuses chandelles ${ }^{11}$, là encore observées sur l'abbatiale, permettent de mieux ajuster les longueurs d'assises, déterminées par le rythme des ouvertures disposées à intervalles réguliers. En effet, les deux piédroits d'une même baie ne comportent parfois pas le même nombre d'assises, offrant ainsi une grande flexibilité dans les choix des moellons; en revanche, les horizontales sont nivelées au niveau des sommiers avant d'amorcer le haut du parement.

Fig. 5 - Façade orientale des Hôtelleries Saint-Hugues (parement est) : relevé archéologique de la moitié nord (dessin C. Gaillard et S. Piotrowski, d'après équipe de fouille).

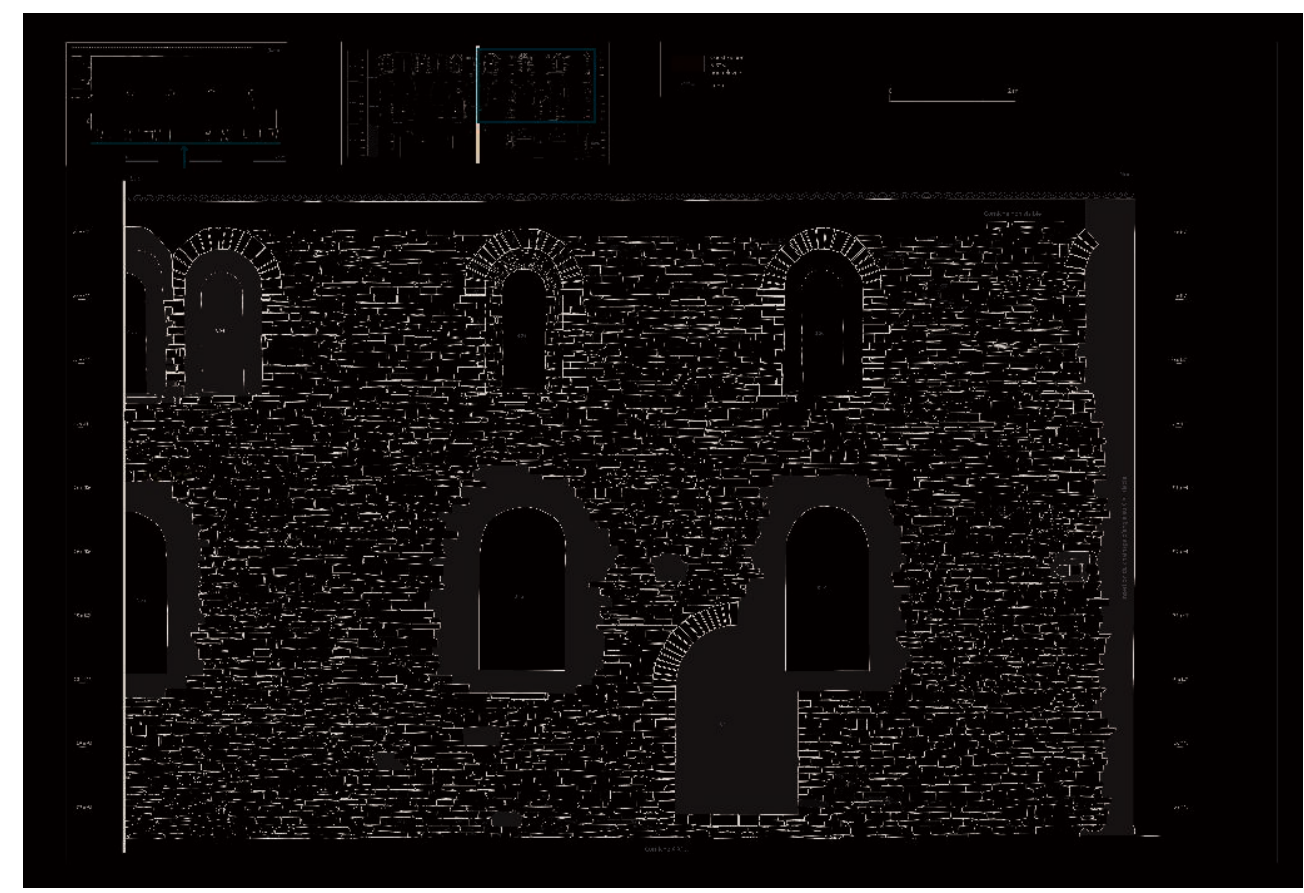

L'édifice est doté de nombreuses ouvertures qui procurent aux deux niveaux une lumière abondante. En partie basse, six petites baies en plein cintre de forme très allongée $(2,70 \mathrm{~m}$ de hauteur par $0,85 \mathrm{~m}$ de largeur) subsistent sur la façade orientale (fig. 1). Très faiblement ébrasées à l'intérieur en raison de leur étroitesse, elles sont espacées de 4 à $5 \mathrm{~m}$ l'une de l'autre. Si l'on reproduit ce même rythme sur la portion manquante du mur, trois baies supplémentaires s'insèrent avant que ne prenne place la dernière ouverture qui subsiste dans la salle Victor Duruy. Le mur pignon sud présentait la même configuration, percé de deux ouvertures disposées de chaque côté 
du pilastre qui amorce le départ de la colonnade ${ }^{12}$. Quant au pignon nord, il pourrait adopter une configuration similaire même si trois baies seulement sont représentées sur le plan Anonyme ${ }^{13}$. Toutes adoptent le même mode de construction : les blocs des piédroits maçonnés sont chaînés avec ceux des ébrasements et sont surmontés d'un petit arc en plein cintre, formé à l'extérieur de trois claveaux très largement trapézoïdaux. Les intrados sont construits en petites dalles disposées de chant, dont l'amorce débute à hauteur constante, régulée par endroits par de petites assises de calage. Certains ont conservé la trace du coffrage, formé de deux rangées de planches de 80 à $90 \mathrm{~cm}$ de long mises chant contre chant, soutenues par un cintre en bois intermédiaire et deux aux extrémités (fig. 6).

Fig. 6 - Baie du rez-de-chaussée comportant des traces de coffrage dans l'intrados (cl. A. Roger).

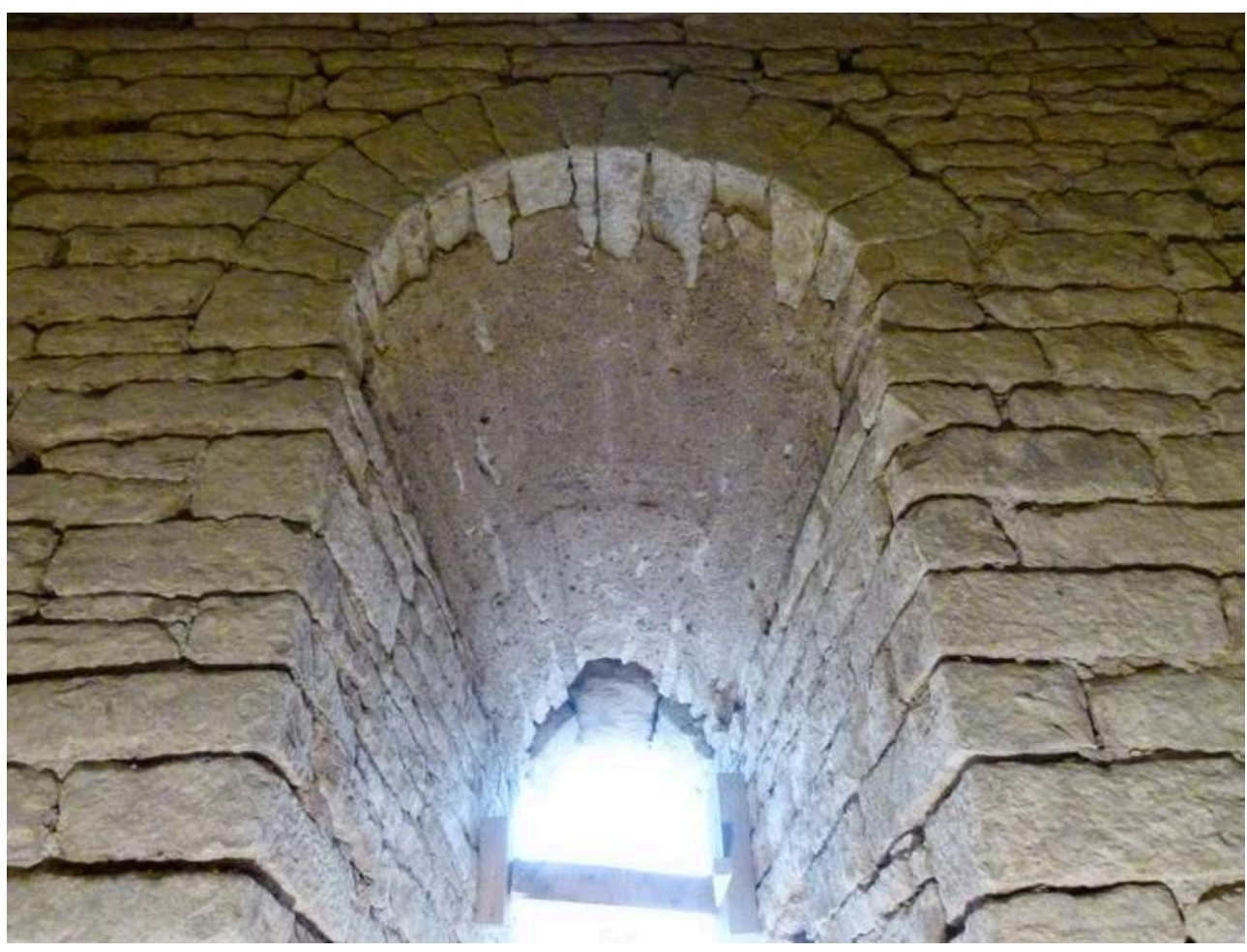

Enfin, ces baies étaient dotées d'un glacis, qui optimisait ainsi au maximum le faible éclairage procuré par les fentes extérieures. Ce mode de construction assure une assise très solide au bâtiment: en effet, les baies rythment la construction de blocs de maçonnerie compris entre les ouvertures, parements internes et externes étant ainsi fermement liés et les assises nivelées par de petits réglages assurés dans les ébrasements.

11 À l'étage, sept baies en plein cintre à double ébrasement rythment la façade orientale, espacées elles aussi de 4 à $5 \mathrm{~m}$ environ : complétées par quatre autres manquantes, elles procurent l'éclairage de la salle haute (fig. 2). Elles se poursuivent sur le mur sud et vraisemblablement sur le mur nord ${ }^{14}$, surmontées ici d'un triplet logé dans le pignon. Elles s'implantent juste sous la corniche, jouant ainsi le rôle d'un clair étage. Récemment démurées en partie, elles ont conservé pour certaines un enduit épais recouvert d'un simple badigeon blanc, sans qu'aucune trace de décor ne soit perceptible. Un système d'accroche de châssis a pu être observé pour l'une d'entre elles: de petits orifices de scellement rythment en effet l'ébrasement intérieur, 
révélant la présence d'un cadre vitré. Par ailleurs, la salle V. Duruy a révélé sur son parement ouest une baie à simple ébrasement $-3,50 \mathrm{~m}$ de hauteur par $1,25 \mathrm{~m}$ de largeur -, située à un niveau inférieur. Or, des traces de badigeon de chaux ornées de fleurs à cinq pétales de couleur rouge, se trouvant sur l'intrados de la baie aujourd'hui bouchée, avaient pu être relevées ${ }^{15}$. Le traitement des baies, on le voit donc, diffère ici de manière importante selon les salles et leurs fonctions respectives. En partie basse, les ouvertures se caractérisent par une fente très étroite et très haute, qui procure certes un éclairage, mais restreint à la pièce. En partie haute en revanche, la lumière abonde.

12 La salle haute est simplement coiffée d'une charpente qui, conformément au constat de K.-J. Conant ${ }^{16}$, est encore dans son état d'origine (fig. 7 et 8). L'absence de plafond et le traitement décoratif des bois montrent que l'intégralité de l'ouvrage était destinée à être visible.

Fig. 7 - Relevé de la charpente, coupes transversale et longitudinale (dessin. X. D’Aire, CEM).

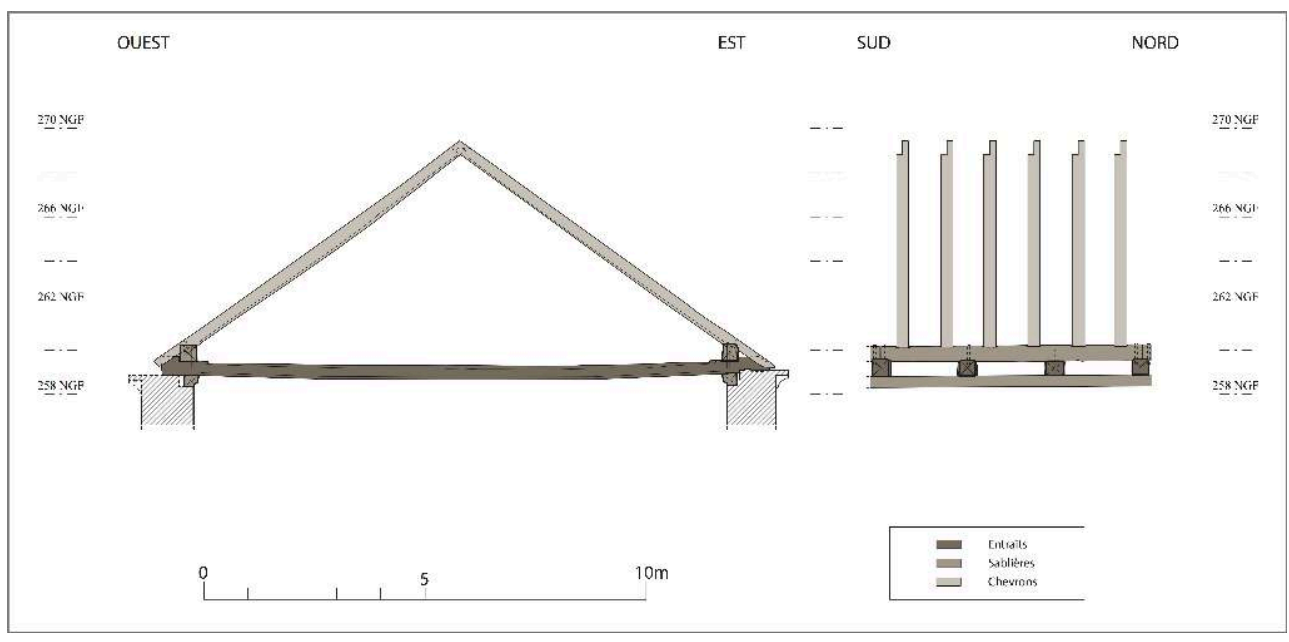


Fig. 8 - Charpente, couple de chevrons nord avec assemblage à mi-bois en tête et arrêtes abattues formant chanfreins et congés (cl. S. Aumard).

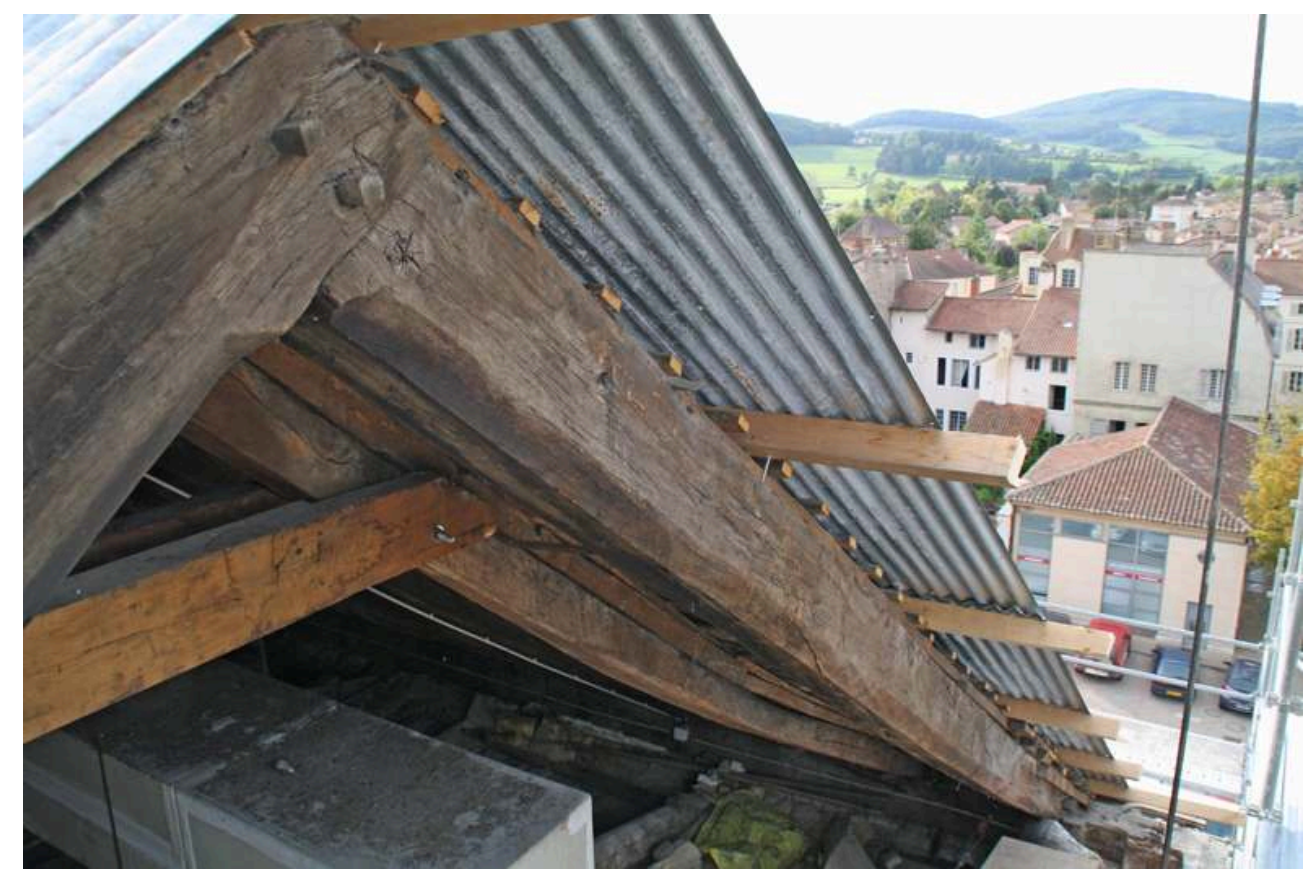

La compréhension de cet ouvrage se trouve favorisée par l'absence de transformation notable, malgré les nombreuses réparations reçues au cours du temps. À la lumière de son étude récente ${ }^{17}$, cette charpente apparaît actuellement comme la plus ancienne de Bourgogne, levée vers 1110 voire dès $1105^{18}$.

Si cette structure partage les caractéristiques des charpentes romanes par la section de ses bois - de 25 à $35 \mathrm{~cm}$, voire $50 \mathrm{~cm}$ - et l'absence de contreventement ${ }^{19}$, sa conception s'en distingue très significativement et fait figure d'unicum à ce jour. Alors que la plupart des ouvrages contemporains intègrent le principe de la triangulation dans le dessin de leurs fermes, en équipant celles-ci d'un entrait en partie basse ou d'un faux entrait en partie haute, la charpente des hôtelleries de Cluny accorde une place très différente à ces pièces horizontales. Ici, les entraits n'appartiennent pas aux fermes, mais à une vaste structure horizontale où ils forment une trame étroite (tous les $2 \mathrm{~m}$ ), et où leurs extrémités sont enserrées entre deux énormes sablières au droit des gouttereaux. Cette trame très robuste reçoit les couples de chevrons espacés de $1 \mathrm{~m}$, simplement assemblés sur la sablière supérieure, sans report de charge.

15 Ainsi, l'édifice se voit doté d'une salle haute de dimension imposante, au traitement particulièrement soigné.

\section{Les galeries extérieures et la question des circulations}

L'analyse archéologique a également permis de réfléchir à la question des circulations internes et externes et de proposer de nouvelles hypothèses. Ces dernières diffèrent en cela des propositions de Kenneth John Conant, qui n'avait manifestement pas eu accès aux maçonneries internes. Tout d'abord, la porte d'entrée principale, large de $2,50 \mathrm{~m}$, est implantée à l'est, rigoureusement centrée dans la façade ${ }^{20}$. Or, un sondage réalisé au niveau du seuil de la porte d'entrée a permis de dégager un premier sol moderne situé à $243 \mathrm{~m}$ NGF et formé d'un dallage de pierre. Quant au niveau médiéval, 
Didier Méhu, qui a fouillé un petit secteur sur la place à l'avant de cette porte, le restitue peu en dessous, à $242,75 \mathrm{~m} \mathrm{NGF}{ }^{21}$. D'après le plan anonyme, une seconde porte s'observe, située sur la façade plus au nord: aujourd'hui détruite, il est cependant vraisemblable qu'elle ait été aménagée lors de la séparation du bâtiment en deux volumes. À l'ouest pour finir, une troisième porte prend place, dont le seuil pourrait se situer également à environ 243 m NGF ${ }^{22}$.

Par ailleurs, les vestiges des deux galeries qui flanquent l'édifice de part et d'autre des façades ont pu être mis en évidence (fig. 4). À l'est tout d'abord, deux portes en plein cintre subsistent en partie à l'étage, aujourd'hui murées, vestiges d'une galerie apposée à l'avant de l'édifice. En outre, deux sondages pratiqués en 1994 ont révélé en fondation deux massifs de maçonnerie, qui débutent à 242,25 m NGF environ et s'implantent à $5 \mathrm{~m}$ environ de la façade orientale. Espacés de 5,50 m l'un de l'autre, ceux-ci sont construits en grandes dalles de $80 \mathrm{~cm}$ de long par $40 \mathrm{~cm}$ de large et $10 \mathrm{~cm}$ d'épaisseur, pris dans un mortier de couleur ocre/rosé à forte teneur en chaux, le tout formant des quadrilatères de $2 \mathrm{~m}$ de côté. L'amorce d'une colonne engagée sur pilier s'observe sur l'une des structures, conservée sur une assise. En parallèle, l'extrémité nord-est du parement de la salle Victor Duruy a révélé en fouille un pilier en saillie de $25 \mathrm{~cm}$ par 1,20 m de large, qui constitue l'extrémité nord de la façade, tandis qu'au sud, le chaînage est en partie refait, trahissant ainsi en négatif le même dispositif. Ainsi, la galerie reposait-elle en effet sur une série d'arcades, ouvertes sur la cour intérieure. Quant au second niveau, les circulations seraient établies aux alentours de $249 \mathrm{~m}$ NGF - soit 6,25 m environ audessus du niveau médiéval supposé ; le mur du rez-de-chaussée est en effet $30 \mathrm{~cm}$ plus large que celui de l'étage, et la saillie ainsi créée, procure l'assise nécessaire à l'installation du plancher de la galerie ; elle est aujourd'hui masquée par une corniche ${ }^{23}$.

Deux éléments de poutre pris dans le parement actuel à 253,80 m NGF témoignent d'un système d'armature implanté dans la fourrure de la façade au moment de l'édification de celle-ci. Disposés perpendiculairement à mi-hauteur de l'étage, ils correspondraient à l'assemblage d'une longrine noyée dans la maçonnerie avec l'about d'un tirant traversant le bâtiment dans sa largeur. Cette ossature devait comprendre également un ancrage similaire dans le mur occidental et formait ainsi une vaste trame rythmée par plusieurs tirants destinée à prévenir de l'écartement des façades ${ }^{24}$.

19 Ainsi, conçue dès l'origine, une galerie s'implante à l'avant de la façade orientale de l'édifice, large de $5 \mathrm{~m}$ environ, et largement ouverte sur la place. Elle restreint donc fortement l'éclairage naturel de la salle basse et justifie la disposition aussi haute des baies, situées au-dessus de sa toiture. À la lumière de ces nouvelles données archéologiques, il nous semble en revanche devoir reconsidérer le modèle proposé par $\mathrm{K}$. J. Conant, qui associe à un niveau d'arcades en pierre un étage construit en pans de bois. En effet, lors des discussions engagées durant le colloque, l'importante dimension des piles maçonnées et leur faible entraxe avaient été soulignés : elles constituent, en effet, un dispositif de fondation paraissant disproportionné pour soutenir une simple galerie de bois. Le pendage nord-sud de la topographie observé en fouille peut en partie expliquer la nécessité d'une telle structure. Rien n'interdit, en revanche, de restituer le haut de la galerie entièrement construit en pierre et ajouré d'une claire-voie, selon le même principe que montre le palais du pape Gélase. En outre, un procès-verbal de visite de 1711 indique que la galerie est portée par un mur de 9,75 m de haut ${ }^{25}:$ si l'on se fie au niveau de sol de la porte situé à $243 \mathrm{~m} \mathrm{NGF}$, il faut restituer un mur qui s'élève 
jusqu'à 252,75 m NGF, soit environ $1 \mathrm{~m}$ sous le niveau d'accroche de la toiture de la galerie dans la façade.

À l'ouest, l'accès actuel au théâtre s'effectue par la même porte en plein cintre située à

l'étage. Néanmoins, les vestiges des structures retrouvées en fouille en 1992 sont plus délicats à interpréter. Cinq piles de construction similaires à l'élévation médiévale s'implantent à $10 \mathrm{~m}$ en avant de la façade; elles semblent s'aligner avec un mur en retour vers le pignon nord de l'édifice. En outre, elles sont de dimension légèrement inférieure à celles de l'est et réparties selon un entraxe de $6,25 \mathrm{~m}$ environ ${ }^{26}$. En revanche, à $5 \mathrm{~m}$ de la façade, une maçonnerie a pu être observée dans deux sondages effectués à chaque extrémité du bâtiment. Au sud, l'élévation semble similaire à la construction des piles. Au nord, cependant, la fondation subsiste seulement en négatif, mais semble former un retour vers un pilier d'angle en saillie sur le même principe que le dispositif observé sur le mur gouttereau est.

21 Ainsi donc, il semblerait qu'une première structure en appentis aurait été aménagée à l'ouest - galerie murée ou ouverte -, doublée ensuite d'une seconde structure, sans doute plus légère - type auvent d'après $\mathrm{N}$. Roiné -, ce qui expliquerait les différences de proportion des piles. Néanmoins, ces données sont à considérer avec prudence et mériteraient d'être réexaminées au moyen de nouveaux sondages archéologiques. La galerie aurait été détruite durant les $\mathrm{XIV}^{\mathrm{e}}$-XV $\mathrm{X}^{\mathrm{e}}$ siècles, en raison des remblais qui scellent les niveaux datés de cette époque. Or, N. Roiné avait mis en évidence un pendage important des niveaux archéologiques de la cour à l'ouest - 7,5\% de pente nord-sud -, qui pourrait avoir entraîné des problèmes de stabilité, ce qui expliquerait peut-être cette destruction précoce.

Au sud, pour finir, une petite annexe s'implante dans l'angle sud-ouest de l'édifice, dont les départs des murs gouttereaux sont chaînés au mur pignon sud. Large de 4,90 m, il n'en subsiste que l'amorce, le reste de la construction ayant été très remanié par le voûtement moderne. L'annexe occupe cependant l'espace d'un vaisseau du bâtiment. Aucune communication n'apparaît avec la salle basse, dont le mur s'ouvre à cet endroit de deux baies étroites. En partie haute, l'élévation de la maison moderne empêche toute observation ${ }^{27}$.

L'analyse de la façade a révélé quelques remaniements ultérieurs, liés à l'implantation de l'aile sud, connue sous le nom d'Hospices de Pierre le Vénérable. La porte située tout au sud, qui ouvrait sur la galerie, est murée et déplacée plus au nord, remplacée par un conduit de cheminée.

\section{Conclusion}

Ainsi, à l'issue de ces observations archéologiques, les contours du bâtiment des Hôtelleries de l'abbaye de Cluny se précisent. Nommé palatium dans le Liber Tramitis, voire peut-être aula magna dans la chronique clunisienne ${ }^{28}$, il constitue un très bel exemple de salle d'apparat médiévale du XII ${ }^{\mathrm{e}}$ siècle. En effet, sa disposition en deux niveaux est classique pour l'époque, qui associe régulièrement espaces de stockage et cuisine au rez-de-chaussée et espace d'hébergement/réception en partie haute. De plus, le traitement architectural et décoratif de la salle haute révèle un espace monumentalisé. L'état originel de la charpente se reconnaît en effet grâce au soin accordé à son traitement décoratif ${ }^{29}$. Des chanfreins et congés rehaussés de rouge ornent systématiquement les arêtes des chevrons et des entraits. Alors que la sablière 
inférieure a reçu une mouluration des plus simples avec cavet et face en dévers, la sablière supérieure et la maçonnerie intermédiaire ont été recouvertes d'un enduit peint de rosaces colorées. Conservé dans un état extrêmement lacunaire ${ }^{30}$, cet enduit semblait assurer sur toute la longueur du bâtiment la continuité du décor apposé aux murs avec celui de la charpente, intégralement visible de l'étage des hôtelleries. Le rôle de cet ouvrage dans la composition monumentale des espaces intérieurs, et notamment de l'étage, ne fait aujourd'hui aucun doute. En outre, s'ajoute la claire-voie haute qui procure à l'édifice un éclairage abondant. À l'ouest en revanche, il est à noter l'absence de baies au rez-de-chaussée et à l'étage, seules trois portes assurant les circulations avec l'extérieur : l'édifice se tourne ainsi résolument vers la place et l'abbaye, opposant donc un mur aveugle à l'entrée du monastère. En outre, les matériaux et leur mise en œuvre semblent assez différents entre les deux murs gouttereaux. Ces observations posent à nouveau la question de la nature des structures situées à l'ouest et de leur rôle dans l'édifice.

Qu'en est-il de la fonction de cet étage "monumental»? Outre le rôle de pièces d'apparat, l'on peut se demander si les différents lieux cités dans le Liber Tramitis ne seraient pas ici condensés en un seul espace - logement des hommes et des femmes, réfectoire et réception des hôtes, séparés par des murs de refend ou des cloisons intérieures. Ceci expliquerait entre autres la présence des différentes portes à l'étage, ainsi que le traitement décoratif différent de certaines parties de la charpente au bas Moyen Âge. Quant aux latrines, aucune structure n'a pu être identifiée : la présence des baies en partie basse de l'annexe sud exclut en effet toute interprétation de ce type ; mais elles pouvaient très bien s'implanter à l'ouest, intégrées à la structure occidentale. De même, aucun conduit de cheminée n'a pu être observé, ce qui renforce également la question des équipements de confort. La présence du clair étage exclut un conduit pris dans l'épaisseur de la maçonnerie et là encore, seul le mur ouest pourrait convenir à ce type de dispositif.

Quant à la galerie latérale qui est aménagée à l'est, elle est prévue dès le départ, avant même la mise en place des Hospices de Pierre le Vénérable. Elle n'assure donc à l'origine aucune communication avec d'autres corps de bâtiments ${ }^{31}$, comme cela peut s'observer dans d'autres lieux, mais joue avant tout un rôle de prestige et d'apparat, dominant la cour et assurant une vue prisée sur le cloître et l'abbatiale. Les ailes de la cour ont été aménagées progressivement : citées dans le dénombrement de 1627, elles sont attribuées à Pierre le Vénérable (hospice) et à la venue du pape Gélase à Cluny (palais du pape Gélase). Certes, l'édification de ces bâtiments s'est étalée dans le temps et pourrait expliquer leur construction progressive. Néanmoins, les observations archéologiques ont pu démontrer que l'aile sud n'est pas prévue dans la conception initiale et que son implantation contraint à des réaménagements au sein de l'hôtellerie : alors condamnée par la nouvelle construction, la porte de l'étage la plus au sud est déplacée plus au nord, tandis que la baie du rez-de-chaussée est transformée en porte et assure ainsi une communication avec le bâtiment sud. La configuration générale de la place correspond cependant au plan général de la réforme clunisienne, qui ménage une place privilégiée aux laïcs, leur réservant ici une cour entière reliée à l'avant-nef de la nouvelle abbatiale Cluny III. 


\section{NOTES}

1. D. IOGNA-PRAT, Ordonner et exclure, Paris, 1998.

2. A. BAUD et G. ROLLIER, "Liturgie et espace monastique à Cluny à la lecture du "liber tramitis", "descriptionemonasterii" et données archéologiques ", in A. BAUD (dir.), Espace ecclésial et liturgie au Moyen Âge, Lyon, 2010, p. 27-42. Le Liber Tramitis évoque les dispositions observées pour Cluny II, mais qui sont reprises pour Cluny III.

3. Connue depuis les travaux de K. J. Conant, cette charpente a fait l'objet de relevés par le Centre de recherche sur les Monuments historiques (Paris) dès le début des années 70, complétés ensuite au milieu des années 90 . Voir infra note 17.

4. K. J. CONANT, Les églises et la maison du chef d'ordre, Macon, 1968, p. 72-73.

5. L'étude archéologique a été menée par C. Gaillard pour le CEM en collaboration avec l'université Lumière Lyon 2. Elle a porté sur le relevé archéologique des murs est (parement extérieur) et sud (parement intérieur) de la partie sud de l'édifice ; la portion nord conservée est aujourd'hui entièrement enduite. L'étude a bénéficié de l'analyse parallèle menée sur la charpente par S. Aumard (CEM), en collaboration avec l'entreprise CEDRE (C. Perrault). Elle s'inscrit dans un travail universitaire de Master 2 plus large mené par S. Piotrowski, intitulé La place des laïcs au sein de la communauté clunisienne. Exemple de l'hôtellerie Saint-Hugues à Cluny, soutenu à l'université Lumière Lyon 2 (dir. A. Baud) en 2014.

6. D. MÉHU,Cluny, place du 11 août 1944, rapport de sauvetage urgent, Dijon, SRA, 1994 ; N. ROINÉ,Cluny, site de l'ancienne abbaye, 71, galerie ouest des écuries de Saint-Hugues, diagnostic archéologique, Dijon, SRA, 1992.

7. K. J. CONANT, Les églises..., op. cit., p. 72-73.

8. Le travail présenté ici se limite volontairement à une restitution architecturale de l'édifice, une réflexion plus large portée sur l'accueil au sein de l'abbaye étant en cours dans le cadre d'une publication d'envergure sur l'archéologie du monastère médiéval.

9. La structure de ce plancher semble avoir été partiellement renouvelée, mais sa conception d'origine se perçoit facilement. De nombreuses solives ont été remplacées par des chevrons de la charpente romane reconnaissables à leurs chanfreins rehaussés de rouge. L'analyse de plusieurs bois prélevés lors de la restauration du pignon nord, confirme l'importance de ces reprises aux $\mathrm{XVIII}^{\mathrm{e}}$-XIX ${ }^{\mathrm{e}}$ siècles et la vraisemblable contemporanéité de ce plancher avec la charpente du début $\mathrm{du} \mathrm{XII}^{\mathrm{e}} \mathrm{s}$. Ce dernier fait actuellement figure d'un des plus anciens exemplaires connus en Bourgogne avec ceux des celliers de Tournus et de La Charité-sur-Loire ( $\mathrm{xI}^{\mathrm{e}} \mathrm{s}$.) et du palais épiscopal d'Auxerre (v. 1120).

10. A. BAUD, Cluny, un grand chantier médiéval au cœur de l'Europe, Paris, 2003.

11. Pierres de calage de petites dimensions disposées verticalement.

12. Seules trois d'entre elles ont pu être observées archéologiquement, en raison de l'insertion d'une porte.

13. Une petite pièce située entre la porte d'entrée de la cour et l'hôtellerie pourrait en effet avoir obstrué la quatrième baie : en l'absence de vestiges, il n'est pas possible de déterminer si cette salle est construite d'origine ou ajoutée dans un second temps.

14. Le pignon nord, aujourd'hui détruit, s'observe néanmoins sur la gravure de Lallemand, qui semble indiquer le même système d'ouvertures que sur le pignon sud.

15. J. ROLLIER, Rapport d'intervention sur les peintures murales de la fenêtre ouest du bâtiment nord, Dijon, SRA, 1992.

16. K. J. CONANT, Les églises..., op. cit., p. 72. 
17. Pour les études antérieures, se reporter à : B. MAURICE et J.-D. SALVÈQUE, « La charpente de l'hospice de l'abbaye de Cluny ou "Écurie de Saint-Hugues" ", in G.-N. LAMBERT et B. MAURICE Les veines du temps, Autun, sd [1992], p.176; P. GARRIGOU-GRANDCHAMP et J.-D. SALVÈQUe, «Les charpentes et les plafonds des $\mathrm{XII}^{\mathrm{e}}$, XIII ${ }^{\mathrm{e}}$ et XIV ${ }^{\mathrm{e}}$ siècles dans l'architecture civile de Cluny (Saôneet-Loire) ", in Le bois dans l'architecture, Paris, sd [1995], p. 40-41 ; G.-N. LAMBERTet alii, « La datation par dendrochronologie, synthèse des campagnes 1988-1990 ", in P. GARRIGOU-GRANDCHAMP et alii, La ville de Cluny et ses maisons, $\mathrm{XI}^{\mathrm{e}}-\mathrm{XV}^{\mathrm{e}}$ siècles, Paris, 1997, p. 232.

18. La plupart des bois échantillonnés sont abattus au cours des deux automnes-hivers 1107-1108 et 1108-1109. Un arbre coupé au printemps 1107 pourrait éventuellement témoigner d'un démarrage plus précoce du levage de la charpente.

19. Celui-ci est uniquement assuré par la volige.

20. K.-J. Conant, dans ses restitutions, la replace beaucoup plus au sud, détruite par la nouvelle porte élaborée au $\mathrm{XIX}^{\mathrm{e}}$ siècle; invisibles à l'extérieur, les départs des piédroits subsistaient en partie intérieure.

21. Aucun niveau de sol médiéval n'a pu être retrouvé ; en revanche, les fondations de la façade, légèrement débordantes, débutent à 242,65 m NGF.

22. Le seuil se situe aujourd'hui à $243,75 \mathrm{~m} \mathrm{NGF}$, mais les piédroits se poursuivent manifestement sous le sol actuel. D'après les observations de N. Roiné, le niveau de sol contemporain se situerait ici aux alentours de $243 \mathrm{~m}$ NGF.

23. La corniche est vraisemblablement aménagée lors de la destruction de la galerie, afin de protéger le mur en saillie des écoulements des eaux.

24. Cette découverte fait échos aux tirants décrits pour la grande église en 1728 (P. BERNARDI, "Architecture médiévale et sources modernes: l'exemple de l'abbaye de Cluny», Bulletin Monumental, t. 151-III, 1993, p. 494). Plusieurs cas contemporains des constructions romanes ont déjà été mis en évidence en Bourgogne, comme dans les régions voisines (par exemple à Gigny dans le Jura) ainsi qu'en Allemagne et en Italie où de nombreux exemples sont encore visibles de

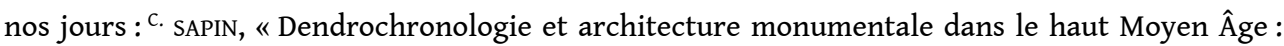
problèmes spécifiques ", in Les veines du temps, op. cit., 1992, p. 159-175; C. SAPIN, S. AUMARD, M. LE BRECH, Gigny-sur-Suran (Jura), église Saint-Taurin, transept et collatéral sud : restaurations des combles, rapport d'étude archéologique préventive, Auxerre, CEM, p. 34; W. HAAS, « Hölzerne und eiserne Anker anmittelalterlichen Kirchenbauten », Architectura, 13, 1983, p. 136-151; T. BELLA, « À propos des armatures en bois dans les églises romanes de l'Italie du Nord: Saint-Ambroise et Saint-Celse de Milan, Saint-Michel de Pavie », Bulletin monumental, 2012, p. 291-308.

25. Le procès-verbal, publié par P. Bernardi, cite un mur de 5 toises de haut; d'après D. Méhu, la toise mesurant $1,95 \mathrm{~m}$, l'on peut restituer en effet un mur de $9,75 \mathrm{~m}$ de haut. P. BERNARDI, «Architecture ... », op. cit., p. 486-493.

26. À l'ouest, les piles adoptent une section de 1,60 x 1,40 m de côté, tandis qu'à l'est, elles avoisinent les $2 \mathrm{~m}$.

27. D'autres remaniements apparaissent sur le mur-pignon sud (trous de poutre, trace de charpente à deux pans, communications), indiquant qu'un édifice est venu s'accoler contre les Hôtelleries. Néanmoins, il n'apparaît pas sur le plan anonyme et peut donc être très récent.

28. K. J. Conant, en effet, précise que d'après les chroniqueurs de la Bibliothecacluniacensis(col. 1669), l'abbé Bertrand de Colombiers (1295-1308) apposerait un nouveau décor aux Hôtelleries : "Item fectichamberium de Cruce, et de magna aula hospitii, et picturisdecoravit. » Néanmoins, en l'absence d'autres indications topographiques, rien ne permet d'affirmer qu'il s'agit bien du même bâtiment évoqué ici.

29. D'après F. Épaud (CNRS), il s'agirait probablement de la plus ancienne charpente décorée qui ait été recensée à ce jour en France.

30. K.-J. Conant en a dessiné plusieurs panneaux, cf. fond Conant au musée Ochier à Cluny. 
31. En revanche, si la salle haute se divise en plusieurs pièces, elle pourrait assurer également des circulations.

\section{RÉSUMÉS}

Les «Hôtelleries Saint-Hugues » furent édifiées durant la grande campagne de construction de Cluny III entreprise par Hugues de Semur (1049-1109). Situé à proximité de la porte d'entrée de l'ancienne abbaye, ce bâtiment s'ouvrait sur une grande cour intérieure qui donnait accès au cloître monastique, bordée au nord par l'avant-nef et au sud par l'aumônerie. Ce bâtiment n'avait finalement fait l'objet que de peu d'investigations jusqu'alors, hormis la solide reconstitution proposée par Kenneth John Connant lors de son travail sur l'abbaye. En 2012, le projet de restauration des élévations orientales et méridionales de l'édifice a ainsi permis de réaliser une étude archéologique sur le bâti concerné, fortement remanié au XIX siècle, accompagné d'une analyse fine de la charpente, appuyée de datations par dendrochronologie. L'étude, confrontée aux résultats issus de divers sondages effectués à l'avant et à l'arrière des bâtiments dans les années 1990, ainsi qu'aux sources graphiques et archivistiques, conduisent à proposer une restitution de l'édifice primitif, remarquable tant par l'ampleur de ses dimensions que par le soin apporté à sa construction.

The « Guest-house of St Hugh of Cluny » was built during the construction of Cluny III by Hugh of Semur (1049-1109). It was situated near the abbey's entrance and opened on a large courtyard giving access to the cloister, between the front nave on the north side and the chaplaincy on the south side. This building remained quite unknown despite Kenneth J. Connant's important study. In 2012, a restoration project regarding the eastern and southern façades allowed an archeological study of the building, wich was rearranged during the XIX ${ }^{\text {th }}$ century, and a study of the framework using dendrochronology. These new results were then confronted to those of several diggings made in the front and in the back of the building during the 1990's and to other sources (archives and drawings). It permits to outline an impressive large and well-constructed primitive building.

\section{INDEX}

Index géographique : France/Cluny

Mots-clés : hôtellerie, archéologie du bâti, monachisme

\section{AUTEURS}

\section{SYLVAIN AUMARD}

Centre d'études médiévales, Université de Bourgogne, UMR Artehis

\section{CHARLOTTE GAILLARD}

Université Lyon 2, Archéologie et archéométrie 


\section{SANDRA PIOTROWSKI}

Université Lyon 2, Archéologie et archéométrie 